\title{
An Efficient Approach of Artificial Neural Network in Runoff Forecasting
}

\author{
Satanand Mishra \\ Scientist \\ CSIR-AMPRI, Bhopal
}

\author{
Prince Gupta \\ PG Scholar \\ VNS Group, RGPV
}

\author{
S.K.Pandey \\ Astt. Professor \\ VNS Group,RGPV
}

\author{
J.P.Shukla \\ Principle Scientist \\ CSIR-AMPRI, Bhopal
}

\begin{abstract}
This survey paper focused on data mining technique based on artificial neural network and its application in runoff forecasting. The long-term and short- term forecasting model was developed for runoff forecasting using various approaches of Artificial Neural Network techniques. This study compares various approaches available for runoff forecasting of artificial neural networks (ANNs). On the basis of this comparative study, it is tried to find out better approach in perspective of research work.
\end{abstract}

Keywords: Data mining, Artificial neural network, forecasting, wavelet analysis, SOM clustering.

\section{INTRODUCTION}

Data mining is use to extract vital information, hidden patterns from huge and distributed database. Extracting knowledge from these databases, will use further for decision support system or to make predictions. Hydrological prediction is one of the important and major domains of hydrological science, where data mining techniques can be implemented to improve the productivity of its analysts tremendously by transforming their voluminous, unmanageable information into usable pieces of knowledge [9]. Streamflow forecasting is a necessary in respect of operation and optimization of water resources. Hydrological data warehouse have record of daily water level, river discharge, rainfall, temperature, humidity, snow melt, evaporation and precipitation data. Forecasting and prediction are made on the basis of these hydrological historical data. This is non-linear and linear time series data. Time series data mining is the combination of data mining with time series analysis for extracting features for building improved predictive model. Artificial neural network (ANN) use the concept of nonlinear mapping and it is useful when the information given is incomplete and noisy where rules cannot be defined. Artificial neural networks (ANNs) have strong capability of pattern reorganization and classification from their learning experience. Apart from ANN, the other models are either mathematical or statistical. These models have been found to be very accurate in calculation, but not in prediction as they cannot adapt to the irregularly varying patterns of data which can neither be written in form of a function, nor deduced from a formula [10]. These real-life situations have been found to be better interpreted by artificial neurons which learn from experience. In this paper key technology and ways to achieve hydrological forecasting pattern using neural networks and their scope are researched in details.

\subsection{Motivation}

In present research, an attempt has made for hydrological forecast by Artificial Neural Network Approach. This research focused on various Artificial Neural Network approach and their potential as well as limitations. Based on existing work, proposed a new approach for hydrological forecast.

\section{APPROACHES OF ARTIFICIAL NEURAL NETWORK IN HYDROLOGICAL PREDICTION}

Forecasting is one of the crucial research topics in the analysis of hydrological time series data. In this sequence to improve the future prediction accuracy of water level and runoff in Indian river system, this survey paper focused on different techniques of ANN. On the basis of literature survey, Artificial Neural Network techniques used in hydrological forecasting in the three forms:

\section{Neural Network Based on Back Propagation \\ 2. Regression Based Neural network \\ 3. Wavelet Neural Network Based Approach}

All the researchers have determined the accuracy of prediction of their proposed ANN approach in the form of Coefficient of determination $\left(\mathrm{R}^{2}\right)$, mean squared error (MSE), mean relative error (MRE). This is the more suitable criteria of accuracy evaluation/.

\subsection{Neural Network Based on Back Propagation Approach}

Backpropagation is a common method to train an Artificial Neural Network how to perform a given task. The objective of training is to find the set of weights between neurons that determine the global minimum of error function. The Backpropagation algorithm is used in layered feed forward ANNs. Signals are forwarded from one layer to other layer and errors are propagated backwards. In this learning process, the algorithm with examples of the input and outputs want the network to compute, and then error is calculated. The main idea of the Backpropagation algorithm is to minimize this error, until the ANN learns the training data.

Abhishek et. al. estimated average rainfall using Backpropagation algorithm along with the multilayered artificial neural network. By using artificial neural network model two phases of learning cycle, one to propagate the input pattern through the network and other to adopt the output by enhancing the weights in the network have been analyzed. It is found that as the number of neurons increases in an ANN, the MSE decreases and BPA is the best algorithm out of the three tested [1].

In this study Adamowski, J. and Karapataki, C. compared three different type artificial neural network and multiple linear regression model for peak weekly water demand forecasting. 20 Levenberg-Marquardt artificial neural network models, 20 resilient back-propagation ANN models, and 20 conjugate gradient Powell Beale ANN, 20 multiple linear regression models, models were developed and their relative performance was compared. This study indicated ANN 
method was a more accurate prediction method with respect to other conventional data mining approaches [2].

In this paper Adamowski compared multiple linear regression, time series analysis and artificial neural networks as techniques for peak daily water demand forecast modelling. Analysis has been done over thirty-nine multiple linear regression model, nine time series model and 39 ANN models of their relative performance [3]

In this study Agarwal, A., and Singh, R. D. have developed a Multi-layer back propagation artificial neural network (BPANN) model to simulate rainfall-runoff process. The gradient descent optimization technique was used to develop BPANN model, considering three time scale and unclear dataset. Performance of BPANN is compared with linear transfer function model and result come from comparison shows that proposed model perform better than LTF model [7]

In this there is an emerging field of research characterized by a wide variety of techniques for the application of rainfallrunoff modelling and flood forecasting using ANN has been discussed. This review underline the need for clear guidance in current modelling practice, as well as the analogy of ANN methods with more conventional statistical models [13].

A depth discussion of the development and application of artificial neural networks to flow forecasting in two flood prone UK catchments using real hydrometric data. The results obtained for validation forecasts were of comparable quality to those obtained from operational systems for the River Amber. The strength of the ANN to adjust with missing/nullified data and to "learn" from the event currently being forecast in real time makes it an appealing alternative to conventional flood forecasting models [14].

Devi, C. J. et. al. present a neural network-based algorithm for predicting the temperature. A feed forward neural network trained with back propagation algorithm is used for temperature forecasting. The proposed model is tested on real time dataset and its performance was also compared with practical working of meteorological department. Extracted result based on comparison shows that proposed model have ability for wealthy temperature forecasting [15].

Edossa and Babel have been modeled a nonlinear stream flow forecasting modeling technique along with ANN for study the drought forecasting. In this available dataset was divided into two independent sets using a split sampling tool of neural network software. The stream flow predicted using the model on weekly time step compared with the major stream flow during the validation period [17].

A time saving and accurate forecasting system with adjustable parameters has been described in this research paper. A regression analysis for water flow forecasting using neural networks has been implemented for Dams, river basin and Proposed method performance has been compared under given parameters [18].

The study of Hsieh et. al. investigated the prediction system with single-point river stage, upstream-downstream river flow forecasting, and rainfall-runoff hydrological process. The prediction for river flow can be obtained by generating the relation between training length and performance parameter. Implemented algorithm can be used to simulate the influence of river flow and stage prediction of Mississipi River [19]

This research contributed in the design, implementation and comparison of rainfall forecasting models using Focused
Time-Delay Neural Networks (FTDNN). Training and testing were performed on the datasets and corresponding accuracies of the forecasts were measured using mean absolute present error [20].

Kumar and Yadav have been introduced ANN as one of most important modern tool used for data processing and hydrological forecasting. In this research feed forward neural network trained with Levenberg-Marquardt back propagation algorithm for rainfall forecasting. The regression coefficient was measured for accuracy of developed model [23]

Kumarasiri, A. D., and Sonnadara, U. J. have been attempted to implement both short term and long term forecasting with the help of feed forward back propagation artificial neural network for daily occurrence and annual depth of rainfall at a single meteorological station. Two neural network models were developed and trained with back propagation algorithm for one day ahead and one year ahead prediction [24]

Luc, K. C. et.al. presented the results of a study investigating the application of ANNs to forecast the spatial distribution of rainfall for an urban catchment. Artificial neural network technique is used for rainfall forecasting. Three different types of ANNs (MLFN, PRNN, and TDNN) were developed and their performances are compared. All three networks had comparable performance when they were developed and trained to reach their optimal complexities [25].

To resolve the parameter calibration which is very tedious and subjective in many cases an alternative conceptual models along with empirical models which relate input and output by mean of an arbitrary mathematical function that bear no direct relationship to the physical characteristics of rainfall-runoff process has been developed. This research evaluated the capacity of ANNs to model with accuracy the monthly rainfall-runoff process [26]

A survey of 43 papers handling with the implementation of neural network models for the forecasting and prediction of water resources variables is undertaken with respect of the modelling process adopted. The large majority of these networks were trained using the back propagation algorithm. Issues in relation to the optimal division of the available data, data pre-processing and the choice of appropriate model inputs are seldom considered. Based on the process obtained result of ANNs have the potential to be a useful tool for the prediction and forecasting of water resources variable [27]

Minns,A.W. and Hall, M.J. developed a ANN model for rainfall runoff forecasting. ANN model is trained with back propagation algorithm. A number of numerical analysis and experiments, in which flow data were generated from synthetic storm sequences routed through a conceptual hydrological model consisting of a single nonlinear reservoir, has described the closeness of fit that can be carry out to such data sets using Artificial Neural Networks (ANNs). The application of different standardization factors to both training and verification sequences have underlined the importance of such factors to network performance [28]

Mishra, S. et. al. presented the analysis based on data mining technique in hydrological daily discharge time series of the panchratna station in the river Brahmaputra under Brahmaputra and Barak Basin Organization in India. Kmeans, Dynamic Time Wrapping (DTW), and agglomerative hierarchical clustering are used to cluster and discover the discharge pattern in terms of the modelling [29]. 
Mittal et. al. proposed to develop a Dual Artificial Neural Network to improve the model performance in terms of extreme values. Proposed model performance compared with feed forward ANN and extracted result indicate that D-ANN model perform well than FF-ANN model [30].

Mutlu et. al. study presented an evolution to use of ANN models to forecast daily flows at multiple gauging station in Eucha watershed. In this study the MLP and radial basis feed forward neural network (RBFNN) were developed and their abilities to predict stream flow were compared [31].

This paper investigated the use of artificial neural networks in the area of synthetic inflow generation. The several steps involved in the development of a neural network and a multivariate autoregressive model for synthesis. The application of both types of model for synthesizing monthly inflow records for two reservoir sites is described. The performance analysis of the neural network is compared with the statistical technique of synthetic inflow generation [33].

In this research Riad et. al. has been developed an ANN model to analyze the rainfall runoff relationship. Multilayer perceptron neural network based model indicated that ANN method is more confined to predict river runoff then classical regression model [35].

Archana and Rakesh examined the study to model the event based rainfall runoff process. A case study has been done to develop event based rainfall runoff model for the basin to simulate the runoff. Obtained result demonstrates that ANN model are able to provide a good simulation for event based forecasting [37]

A performance comparison of three artificial neural network techniques for adding simulated river flows, based on three different neural network structures has been done. These network structures are: the radial basis function neural network (RBFNN), the simple neural network (SNN), and the multilayer perceptron neural network (MLPNN). The result of the study showed that performances of all three combination methods are, on average, better than that of the best individual rainfall-runoff model utilize in the combination [38].

In this research Sharda et. al. analyzed three hilly micro watersheds to assess the impact of morphological characteristics on water flow. To predict runoff and base flow, an artificial intelligence model is developed using multivariate adaptive regression splines method. The predicted value from proposed model found good and it is observed that MARS model have good capability of simulate runoff in hilly area [39].

This paper compared different training technique available for training multilayer perceptron type of artificial neural networks for modeling the rainfall runoff process. The training method includes the back propagation algorithm (BPA), real coded genetic algorithm (RGA) and a Self organizing map (SOM). A wide variety of standard statistical performance evaluation measures were employed to evaluate the performance of various ANN models developed [41].

$\mathrm{Wu}$ et. al. demonstrated an application of ANN for runoff and stream flow forecast. This research has been the development of the development of methodology for input data organization, model performance evaluation, and ANN processing techniques. Two ANN model have been developed and trained with back propagation algorithm for near-realtime and near-term-flow predictions. Results obtained indicate that ANN-hydrologic forecasting models can be considered an alternate and practical tool for stream-flow forecast [44].

\subsection{Regression Based Neural network Approach}

The general regression neural network (GRNN) is a one-pass learning algorithm with a highly parallel structure [39]. The GRNN is used for estimation of continuous variables, as in standard regression techniques. It is related to the radial basis function network and is based on a standard statistical technique called kernel regression. The algorithmic form can be used for any regression problem in which an assumption of linearity is not justified.

Cigizoglu, H. K. employed an ANN algorithm, generalized regression neural network (GRNN), in monthly mean flow forecasting. The performance of GRNN is compared with the feed forward back propagation (FFBN) method. Observed data (based on comparison) used to train the neural network and forecasting is carried out using AR model. After compare the model performance based on performance criteria it is found that GRNN perform better than FFBN [11].

In this study Cigizoglu, H. K. and Alp, M. deployed generalized regression neural network (GRNN) for river suspended sediment estimation. The result comes up from ANN algorithms are compared with conventional sediment rating curve and multi linear regression methods result. The mean squared error and the determination coefficient are used as performance criteria. Based on performance criteria it was seen that the GRNN performances superior to feed forward back propagation (FFBP) [12].

In this study, Dreiseitl, S., and Ohno-Machado, L., encapsulate the similarities and difference between logistic regressions, artificial neural network and compared their performance with other conventional machine learning algorithm. On the basis of comparison ANN and logistic regression found better than the conventional machine learning algorithm until newer machine learning algorithms, such as support vector machines and other kernel-based algorithms, can prove to be significant [16].

Koutsoyiannis, D. discussed the research paper Kisi,O.(2006), generalized regression neural network for evapotranspiration modelling. Kişi (2006) compares the ANN results with other existing approximations such as Hargreaves and Ritchie equations. Hargreaves and Ritchie equations do not contain any adjustable parameters whereas ANN contains various adjustable parameters for optimization. Then it cannot be concluded that the GRNN1 model outperforms all other models in terms of various performance criteria [21].

In this study Kisi, O. investigated the ability of generalized regression neural networks technique in evapotranspiration $\left(\mathrm{ET}_{0}\right)$ modelling obtained using the FAO Penman-Monteith (PM) equation. The performance of GRNN model was compared to the empirical model using mean square error, mean absolute error. Result obtained based on comparison shows that GRNN model strongly employed in evapotranspiration modelling [22].

Specht, D. F. describes a new network that provides estimates of continuous variables and converges to the linear or nonlinear regression surface. The proposed memory based general regression neural network (GRNN) features fast learning that does not require an iterative procedure, and a highly parallel structure. It can be used for prediction, modeling, mapping, and interpolating or as a controller. The 
algorithmic form can be used for any regression problem in which an assumption of linearity is not justified. [40]

In this research paper Wang, Z. L. and Sheng, H. H. introduce the application of generalized regression neural network (GRNN) model to forecast annual rainfall. The result of proposed method is compared with the back propagation neural network and regression analysis method. Compared with the traditional linear model and BP neural networks, the GRNN has smaller prediction error. The results and accuracy of GRNN model comes better than that of BP neural network for annual rainfall [42].

\subsection{Wavelet Neural Network Based Approach}

This section present a neural network technique combined with wavelet analysis. Wavelet analysis is employed to preprocess the data [10]. The wavelet decomposition of nonstationary time series into different scales provides an interpretation of the series structure and extracts the significant information about its history, using few coefficients. For these reasons, this technique is largely applied to times series analysis of non-stationary signals.

Adamowski, J. and Sun, K. proposed a method, for flow forecasting applications in non-perennial rivers in semi-arid watersheds which is based on coupling discrete wavelet transforms (WA) and artificial neural networks (ANN) techniques. The discrete wavelet transform is used to preprocess the input data for ANN model by decompose time series data into wavelet coefficients. The wavelet coefficients are then used as inputs into artificial neural network models for flow forecasting. The performance of the coupled waveletneural network models (WA-ANN) was compared to classic artificial neural network (ANN) models and the performance of coupled wavelet-neural network models were found to superior than the artificial neural network models [4].

Adamowski, J. et. al. developed three model; multivariate adaptive regression spline (MARS), wavelet transform artificial neural network (WA-ANN), and regular artificial neural network (ANN) for runoff forecasting applications. MARS model is build using MARS ${ }^{\mathrm{Tm}}$ software (version 2). Regular ANN model is used and Levenberg-Marquardt (LM) algorithm was used to train ANN model in MATLAB. WAANN model are the combination of ANN and wavelet analysis technique. The performances of these models were compared. Result showed that the best WA-ANN and MARS models provide very accurate runoff forecasts compared to the best ANN model [5].

Adamouski, J. and Chan, H. F. come up with a new approach based on pairing discrete wavelet transforms (WA) and artificial neural networks (ANN) for groundwater level forecasting. The performance of the proposed coupled wavelet-neural network models (WA-ANN) was compared to regular artificial neural network (ANN) models and autoregressive integrated moving average (ARIMA) models for monthly groundwater level forecasting. Based on the comparison it was concluded that the WA-ANN models provide more accurate forecasting result compared to the ANN and ARIMA models [6].

In this study Adamowski and Belayneh compared the effectiveness of three data-driven models for forecasting drought conditions in the Awash River Basin of Ethiopia. The Standard Precipitation Index (SPI) is forecast and compared using artificial neural networks (ANNs), support vector regression (SVR), and wavelet neural networks (WN). The forecast result indicated that the wavelet neural network models were the best models for forecasting [8].

In this study Campisi-Pinto, S. et. al. come up with a new hybrid approach which is the combination of waveletdenoising and back-propagation artificial neural networks (ANNs) for urban water demand forecasting. The proposed model performance was compared with non-coupled ANN and Linear Multiple Regression models. The results obtained in this study shows that proposed approach perform better than non-coupled ANN and linear multiple regression model and it was observed that by mean of denoising, variance are reduced in the training-set improve forecasting accuracy [9].

In this study Cannas B. et. al. applied neural network and wavelet analysis to predict the hydrologic behavior of the runoff. In this research paper data is preprocessed for neural network input and output using continuous and discrete wavelet transforms. Proposed approach is compared with ANN. comparison result showed that the networks trained with pre-processed data perform better than the networks trained with undecomposed noisy raw signals [10].

In this study Partal,T. and Cigizoglu,H.K. propose a waveletneural network method to predict the daily precipitation from meteorological data. Wavelet neural network is a integration of discrete wavelet transform (DWT) and artificial neural networks (ANN). The proposed method performance was compared with regular ANN and multi linear regression model (MLR) model. The results obtained in this study indicate that WANN model perform superior to other conventional ANN model or a multi linear regression model [32].

Ramana, R. V. et. al. combine the wavelet technique with Artificial Neural Network (ANN) for rainfall prediction. The time series data of rainfall was decomposed into sub series by wavelet analysis. Appropriate sub-series of the variable used as inputs to the ANN model and original time series of the variable as output. To train the network LevenbergMarquardt Algorithm (LMA) is used. The performance of wavelet neural network (WNN) is compared with the ANN and AR model. Based on comparison, it is concluded that the performance of WNN was much better than ANN and AR models in forecasting the rainfall [34].

In this study Santos and Silva come up with a new wavelet and artificial neural network hybrid model for daily stream flow forecasting. The proposed hybrid model performance is compared with classic ANN model. Result obtained in this study indicates that proposed hybrid model give better forecasting result than the classic ANN model [36].

In this research paper Wei, S. et. al. have been come up with a wavelet-neural network (WNN) hybrid modeling approach for monthly river flow evaluation and based on proposed approach prediction model is developed. This proposed approach combines discrete wavelet multi-resolution decomposition and a back propagation (BP) feed-forward multilayer perceptron (FFML) artificial neural network (ANN). The Bayesian regularization and LevenbergMarquardt algorithm were used to perform the network modelling. The results from WNN hybrid model was compared with single ANN model. The comparison shows that WNN hybrid model perform better than the single ANN model [43]. 


\section{PROPOSED APPROACH}

In this paper, a new hybrid methodology is proposed which consist of three techniques: SOM Clustering, Wavelet Analysis, and Artificial neural Network. SOM Clustering and Wavelet Analysis are used for data preprocessing. An SOMbased clustering method is proposed to select the dominant inputs for the FFNN model. To further enhance the efficiency of the proposed model, wavelet-based (WT) temporal preprocessing is also conducted to specify the important features of process. The outcome of Wavelet analysis fed as input for artificial neural Network which finally predicts forecast result.

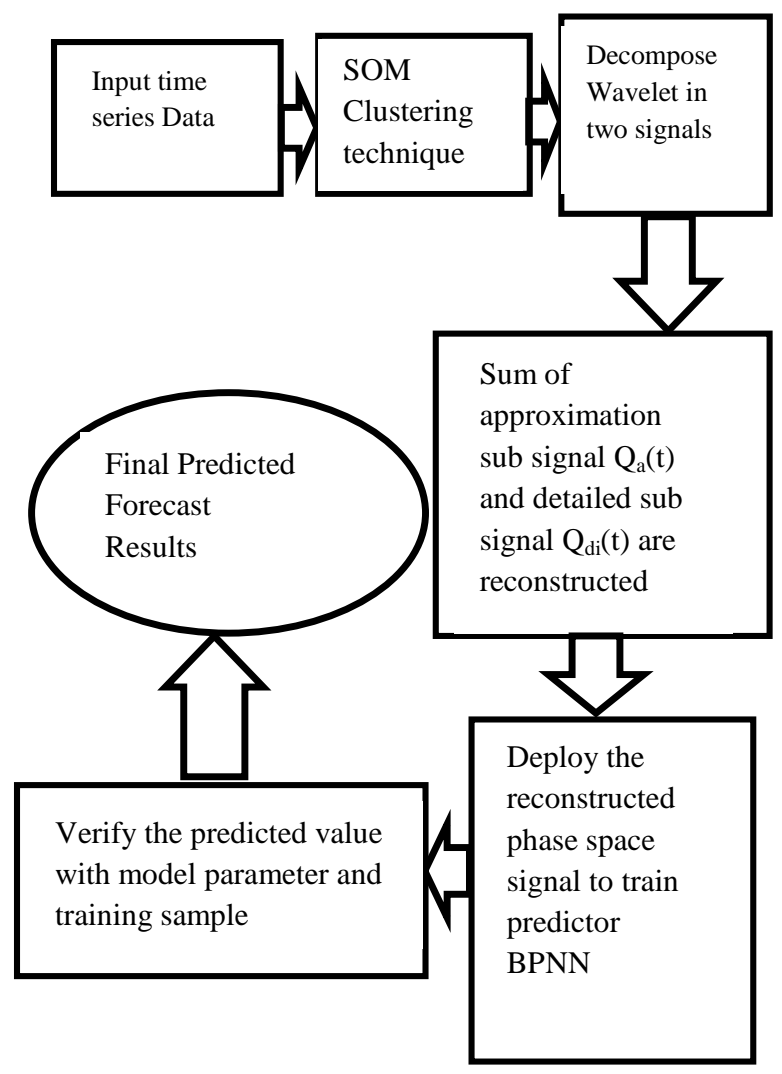

Fig 1: Flow diagram of proposed approach

\section{EVALUATION AND PERFORMANCE ANALYSIS \\ 4.1 Performance Analysis \\ On the basis of literature survey, the performance of approaches is evaluated as under:}

\subsubsection{Neural Network Based on Back Propagation Approach}

A large number of techniques have been deployed on or before implementation of BPNN technique of Data Mining in field of hydrological predictions. However it is unambiguously found that the performance of prediction using BPNN technique is simulating more accurate prediction as compared to traditional approaches.

\subsubsection{Regression Based Neural network Approach}

The majority of the artificial neural network applications in water resources involve the employment of back propagation method. One disadvantage of back-propagation is that it can take a large number of iterations to get to the desired solution. To overcome this problem a new technique is used called
General Regression Neural Network. This general regression neural network (GRNN) is a one-pass learning algorithm with a highly parallel structure. It approximates any arbitrary function between input and output vectors, drawing the function estimate directly from the training data. The forecast of GRNN is bounded by minimum and maximum of observed series. So the training time of GRNN is relatively shorter than BPNN and it enhance the performance of GRNN.

\subsubsection{Wavelet Neural Network Based Approach}

A Wavelet Neural Network Based Approach is the most efficient approach used for hydrological prediction and the main difference from other is that it combines two techniques together; Artificial Neural Network and Wavelet Analysis. Wavelet analysis is used for pre-processed the neural network input. The time series hydrological data was decomposed into sub series by wavelet analysis. Appropriate sub-series of the variable used as inputs to the ANN model and original time series of the variable as output. In the above discussed section so many papers have been studied and generated a detailed view that prediction accuracy is increased if wavelet neural network approach is used.

\subsection{Evaluation Analysis}

The graph is an efficient way of comparing the two types of data available with us. It can also be used to calculate the accuracy of the model. ANN model is quite accurate in prediction. Coefficient of determination $\left(\mathrm{R}^{2}\right)$, mean squared error(MSE), mean relative error(MRE) are used to calculate the accuracy of prediction. $\mathrm{R}^{2}$ measure the linear relationship between two variables.

$$
\begin{gathered}
\text { MSE }=\frac{\sum_{\mathrm{i}=1}^{\mathrm{M}}\left(\mathrm{Q}_{\mathrm{i}}-\hat{Q}_{i}\right)^{2}}{\mathrm{M}} \\
\mathrm{MRE}=\frac{\sum_{\mathrm{i}=1}^{\mathrm{M}}\left|\frac{\mathrm{Q}_{\mathrm{i}}-\widehat{Q}_{\mathrm{i}}}{\mathrm{Q}_{\mathrm{i}}}\right|}{\mathrm{M}} \\
\mathrm{R}^{2}=\frac{\left[\sum\left(Q_{i}-\overline{Q_{i}}\right)\left(\hat{Q}_{i}-\overline{\hat{Q}_{i}}\right]^{2}\right.}{\sum\left(Q_{i}-\overline{Q_{i}}\right)^{2} \sum\left(\hat{Q}_{i}-\widehat{\widehat{Q}}_{i}\right)^{2}}
\end{gathered}
$$

Where $\mathrm{Q}_{\mathrm{i}}$ is observed data, $\overline{\hat{Q}}_{i}$ is arithmetic average of predicted data, and $M$ is total number of observations in either the training or test data set.

\section{RESEARCH ISSUES \& CHALLENGES}

There are some important points that need to be concentrated while hydrological forecasting: performance and reliability of model or timeliness. Some important research issues in this area listed next:

The primary issue of hydrological forecasting is to provide maximum lead time with sufficient accuracy so that users may take appropriate action to mitigate losses or optimize water management decisions.

Various approaches discussed, go into deciding what type of forecasting model should be used. Input data requirements for calibration and operational forecasts vary significantly from model to model.

Due to constant changing of data patterns as well as the presence of noise in data, it is a challenging task to build model for normal prediction behavior.

Due to lack of availability of labeled datasets for training and validation of the models, most approaches in many false 
alarms that require attention. Thus, minimization of false alarm is challenging issues.

\section{CONCLUSIONS}

This paper examined various approaches of artificial neural network for hydrological forecast with their potential as well as limitation and proposed an approach for artificial neural network model building for hydrological forecast. Performance of model structure is evaluated using Coefficient of determination $\left(\mathrm{R}^{2}\right)$, mean squared error (MSE), mean relative error(MRE). The result obtained in the present study has been able to demonstrate that the ANN models are able to provide a good representation of hydrological forecast. The back propagation approach is suitable for above research perspective. Future work is based on simulating and predicting result using back propagation algorithms for runoff forecasting and comparing predicted forecasting result accuracy with current forecasting techniques which ultimately provide a more reliable data mining approach.

\section{REFERENCES}

[1] Abhishek, K., Kumar, A., Ranjan, R., and Kumar, S. (2012), A Rainfall Prediction Model using Artificial Neural Network, IEEE

[2] Adamowski, J. and Karapataki, C. (2010), Comparison of Multivariate Regression and Artificial Neural Networks for Peak Urban Water-Demand Forecasting: Evaluation of Different ANN Learning Algorithms, ASCE

[3] Adamowski, J. F. (2008), Peak Daily Water Demand Forecast Modeling Using Artificial Neural Networks, ASCE

[4] Adamowski, J. and Sun, K. (2010), Development of a coupled wavelet transform and neural network method ofr flow forecasting of non-perennial rivers in semi-arid watershed, ELSEVIER

[5] Adamowski, J., Chan, H. F., Prasher, S. O., and Sharda, V. N. (2012), Comparison of multivariate adaptive regression splines with coupled wavelet transform artificial neural networks for runoff forecasting in Himalayan micro-watersheds with limited data, IWA

[6] Adamouski, J., and Chan, H. F. (2011), A wavelet neural network conjunction model for groundwater level forecasting, ELSEVIER

[7] Agarwal, A., and Singh, R. D. (2004), Runoff Modelling Through Back Propagation Artificial Neural Network With Variable Rainfall-Runoff Data, SPRINGER.

[8] Belayneh, A., and Adamowski, J. (2012), Standard precipitation index drought forecasting using Neural Networks, Wavelet Neural Networks, and Support Vector Regression, Applied Computational Intelligence and Soft Computing Volume 2012 (2012), Article ID 794061, 13 pages.

[9] Campisi-Pinto, S., Adamouski, J., and Oron, G. (2012), Forecasting UrbanWater Demand ViaWavelet-Denoising and Neural Network Models. Case Study: City of Syracuse, Italy, Springer Volume 26, Issue 12, pp 35393558.

[10] Cannas, B., Fanni, A., Sias, G., Tronci, S., and Zedda, M. K. (2005), River flow foreasting using neural networks and wavelet analysis, European Geosciences Union.
[11] Cigizoglu, H. K. (2005), Generalized regression neural network in monthly flow forecasting, Civil Engineering and Environmental Systems, volume 22 issue 2.

[12] Cigizoglu, H. K., and Alp, M. (2006), Generalized regression neural network in modelling river sediment yield, ELSEVIER

[13] Dawson, C. W., and Wilby, R. L. (2001), Hydrological modelling using artificial neural networks, SAGE

[14] Dawson, C. W., and Wilby, R. (1998), An artificial neural network approach to rainfall-runoff modelling,IAHS

[15] Devi, C. J., Reddy, B. S. P., Kumar, K. V., Reddy, B. M., and Nayak, N. R. (2012), ANN Approach for Weather Prediction using Back Propagation, IJETT

[16] Dreiseitl, S., and Ohno-Machado, L. (2002), Logistic regression and artificial neural network classification models: a methodology review, SCIENCE DIRECT

[17] Edossa, D. C., Mukand, S., Babel , Forecasting Hydrological Droughts Using Artificial Neural Network Modeling Technique.

[18] Egawa, T., Suzuki, K., Ichikawa, Y., Iizaka, T., Matsui, T., and Shikagawa, Y. (2011), A Water Flow Forecasting for Dam using Neural Networks and regression models, IEEE.

[19] Hsieh, B. B., Bartos, C. C. L., and Zhang, B. , USE OF ARTIFICIAL NEURAL NETWORKS IN A STREAMFLOW PREDICTION SYSTEM.

[20] Htike, K. K., and Khalifa, O. O. (2010), Rainfall Forecasting Models Using Focused Time-Delay Neural Networks,IEEE.

[21] Koutsoyiannis, D. (2007), Discussion of "Generalized regression neural networks for evapotranspiration modelling, IAHS

[22] Kisi, O. (2006), Generalized regression neural networks for evapotranspiration modelling, IAHS.

[23] Kumar, R., and Yadav, G. S. (2013), Forecasting of Rain Fall in Varanasi District, Uttar Pradesh Using Artificial Neural Network, JECET.

[24] Kumarasiri, A. D., and Sonnadara, U. J. (2008), Performance of an artificial neural network on forecasting the daily occurrence and annual depth of rainfall at a tropical site, Hydrological process, 22, 35353542

[25] Luc, K. C., Ball, J. E., and Sharma, A. (2001), An Application of Artificial Neural Networks for Rainfall Forecasting, ELSEVIER

[26] Machado, F., Mine, M., Kaviski, E., and Fill, H. (2011), Monthly rainfall-runoff modelling using artificial neural networks, Hydrological Sciences Journal, volume 56, issue 3 .

[27] Maier, H. R., and Dandy, G. C. (2000), neural networks for the prediction and forecasting of water resources variables: a review of modelling issues and applications, ELSEVIER

[28] Minns, A. W., and Hall, M. J. (1996), artificial neural networks as rainfall runoff models, Hydrological Sciences Journal. 
[29] Mishra, S., Dwivedi, V. K., Saravanan, C., and Pathak, K. K. (2013), Pattern Discovery in Hydrological Time Series Data Mining during the Monsoon Period of the High Flood Years in Brahmaputra River Basin, IJCA.

[30] Mittal, P., Chowdhury, S., Roy, S., Bhatia, N., and Srivastav, R. (2012), Dual Artificial Neural Network for Rainfall-Runoff Forecasting, Journal of Water Resource and Protection.

[31] Mutlu, E., Chaubey, I., Hexmoor, H., and Bajwa, S. G. (2008), Comparison of artificial neural network models for hydrologic predictions at multiple gauging stations in an agricultural watershed, Hydological Process.

[32] Partal, T., and Cigizoglu, H. K. (2009), Prediction of daily precipitation using wavelet-neural networks, IAHS.

[33] Raman, H. and Sunilkumar, N. (1995), Multivariate modelling of water resources time series using artificial neural networks, Hydrological Sciences Journal, volume 40 , issue 2 .

[34] Ramana, R. V., Krishna, B., Kumar, S. R., and Pandey, N. G. (2013), Monthly Rainfall prediction using wavelet neural network analysis, Springer.

[35] Riad, S., Mania, J., Bouchaou, L., and Najjar, Y. (2004), Rainfalll-runoff model using an artificial neural network approach, ELSEVIER.

[36] Santos, C. A. G., and Silva, G. B. L. (2012), Daily streamflow forecasting using a wavelet transform and artificial neural network hybrid model, Hydrological Sciences Journal.
[37] Sarkar, A., and Kumar, R. (2012), Artificial Neural Networks for Event Based Rainfall-Runoff Modeling, Journal of Water Resource and Protection, 2012, 4, 891897.

[38] Shamseldin, A. Y., O'CONNOR, K. M., and Nasr, A.E. (2007), A comparative study of three neural network forecast combination methods for simulated river flows of different rainfall-runoff models, Hydrological Sciences Journal.

[39] Sharda, V. N., Patel, R. M., Prasher, S.O., Ojasvi, P.R., and Prakash, C. (2006), Modeling runoff from middle Himalayan watersheds employing artificial intelligence techniques, agricultural water management $83,233-2$ 42.

[40] Specht, D. F. (1991), A general regression neural network, IEEE.

[41] Srinivasulu, S., and Jain, A. (2006), A comparative analysis of training methods for artificial neural network rainfall-runoff models, ELSEVIER

[42] Wang, Z. L., and Sheng, H. H. (2010), Rainfall Prediction Using Generalized Regression Neural Network: Case study Zhengzhou, IEEE

[43] Wei, S., Yang, H., Song, J., Abbaspour, K., and Xu, Z. (2013), A wavelet-neural network hybrid modeling approach for estimating and predicting river monthly flows, IAHS

[44] Wu, J. S., P.E., M. Asce., Han, J., Annambhotla, S., and Bryant, S., (2005), Artificial Neural Networks for Forecasting Watershed Runoff and Stream Flows, ASCE 